\title{
Laparoscopic sleeve gastrectomy - 7 years of own experience
}

\author{
Tomasz Szewczyk ${ }^{1}$, Przemyslaw Janczak ${ }^{1}$, Adam Janiak ${ }^{1}$, Tomasz Gaszyński², Bogdan Modzelewski ${ }^{1}$ \\ ${ }^{1}$ Department of Gastroenterology, Oncology and General Surgery, Medical University of Lodz, Lodz, Poland \\ ${ }^{2}$ Department of Emergency and Disaster Medicine, Medical University of Lodz, Lodz, Poland
}

Videosurgery Miniinv 2014; 9 (3): 427-435

DOI: $10.5114 /$ wiitm.2014.44167

\begin{abstract}
Introduction: Laparoscopic sleeve gastrectomy is a procedure frequently chosen by patients and surgeons that carries the risk of serious complications that are difficult to treat.

Aim: To describe the operations performed by us, considering complications and their management.

Material and methods: We performed 565 laparoscopic sleeve gastrectomies. Standard surgical technique was used. A $34 \mathrm{Fr}$ calibration tube was used. An additional reinforcing suture was applied over the staple line.

Results: There was no need for conversion. In $7.79 \%$ of patients, infarcts of the posterior pole of the spleen were observed, whereas 8 patients (1.42\%) developed gastric fistulas in the His angle region. In 3 cases, it led to development of an abscess in the posterior splenic pole region and 2 of these developed secondary gastric fistulas of typical location. In total, there were 5 deaths among the patients who had been operated on - 3 due to septic complications in the course of fistula, 1 due to encephalopathy and 1 as a result of myocardial infarction.

Conclusions: Sleeve gastrectomy is an effective and safe method of obesity treatment. The causes of the most severe complication - gastric fistula - cannot be established unequivocally. Infarcts of the posterior pole of the spleen, as a potential cause of fistulas, deserve particular attention. In our opinion, primary closure of the fistula by suturing is an inappropriate method of management, whereas the best results are obtained with temporary gastrointestinal tract prosthesis.
\end{abstract}

Key words: sleeve, leak, stent, complications.

\section{Introduction}

Laparoscopic sleeve gastrectomy (LSG) has been regarded as a complete bariatric procedure for a number of years so far. Patients and surgeons choose this procedure because of its high effectiveness. Unfortunately, this method is related to a number of serious complications, including death. It has proven to be extremely difficult to standardize the method of management in case of complications due to the small number of operations as well as complications [1, 2].

\section{Aim}

The aim of the work is to describe the operations performed by us, considering complications and their management.

\section{Material and methods}

From October 2006 to December 2013, 565 LSG procedures were performed in the Lodz Medical University's Department of Gastroenterology, Oncology and General Surgery. The patient group consisted of 414 women (73.27\%) and 151 men (26.73\%). The

\section{Address for correspondence}

Tomasz Szewczyk MD, PhD, Department of Gastroenterology, Oncology and General Surgery, Medical University of Lodz, 22 Kopcińskiego St,

91-153 Lodz, Poland, phone: +48 501040 081, e-mail: tomasz.szewczyk@umed.lodz.pl 
patients' average body weight was $134.8 \mathrm{~kg}$ (range: 90-240 kg) and body mass index (BMI) $47.16 \mathrm{~kg} / \mathrm{m}^{2}$ $\left(34.95-65.26 \mathrm{~kg} / \mathrm{m}^{2}\right)$. Detailed demographic data are presented in Table I.

The patients were selected for the procedure according to the principles adopted by International Federation for the Surgery of Obesity and Metabolic Disorders (IFSO). Prior to the procedure, each patient underwent basic blood tests, chest X-ray, thyroid hormone levels assessment, upper gastrointestinal tract (GIT) endoscopy, and psychological consultation, and completed a questionnaire concerning the history of obesity, current health condition, concomitant diseases, and past surgical procedures. Patients who failed to obtain a favorable opinion of the psychologist concerning their understanding of the nature of the procedure and guarantee of cooperation with the medical team in the postoperative period were denied surgery. The procedure was postponed in patients with specific contraindications, such as inflammatory conditions. They were subjected to appropriate treatment and operated on after regression of the disease.

In 16 patients (2.83\%) surgery was performed as a repair procedure after previous bariatric operations, due to lack of effectiveness of previous procedures, or recurrence of obesity. The structure of that group is presented in Table II.

The procedures were performed by one surgical team. The standard surgical technique was used. Dissection of the greater curvature of the stomach was performed with a harmonic knife or Ligasure, beginning in the area located half way across the stomach body. After opening of the omental sac, the stomach was dissected towards the His angle, closing the short gastric vessels and revealing the left portion of the diaphragm. Then the greater curva- ture was dissected towards the pylorus, terminating the dissection about 5-6 cm from it. A 34 Fr gastric tube was introduced into the patient's duodenum through the oral cavity. It was used as a calibration tube for the "new" stomach formation. Ethicon or Covidien linear staplers with $60 \mathrm{~mm}$ long cartridges were used during stomach resection. The green one was always the first to be used, then, depending on the tissue thickness, the green, golden or blue ones. Staples from the last cartridge were applied leaving a margin of about $5-10 \mathrm{~mm}$ from the His angle. An additional continuous suture covering the staple line (prolene 2.0 or $\mathrm{V}$-loc 2.0) was always applied. A latex drain was placed in the His angle region and brought out through one of the trocar insertion sites. A leak test was performed using methylene blue administered under pressure through a gastric tube placed in the region of the gastroesophageal junction. In the first 200 patients, a gastric tube was inserted through the nose down to the proximal level of $1 / 3$ of the reduced stomach. The tube was left in place for 4 days. In the rest of the patients, the tube placement was abandoned. The patients remained in the intensive care unit for the first 24 postoperative hours. They were mobilized $6 \mathrm{~h}$ after completion of the procedure. On the fourth postoperative day, the leak test was repeated with methylene blue administered orally and assessment of abdominal drainage color; then, fluids could be administered to the patient. If there was suspicion of a leakage, contrast abdominal radiography scan was performed. If there had been no leak detected, the drain was removed. Typically, the patients were discharged home on the $5^{\text {th }}$ day after the surgery. On discharge, they received recommendations regarding further diet and care. At 6-month intervals, their body weight loss was mon-

Table I. Demographic data

\begin{tabular}{|lccc|}
\hline Parameter & Females $(N=414)$ & Males $(N=151)$ & Total $(N=565)$ \\
\hline Body weight $[\mathrm{kg}]$ & $129.7(90-190)$ & $158.1(112-240)$ & $134.8(90-240)$ \\
\hline BMl $\left[\mathrm{kg} / \mathrm{m}^{2}\right]$ & $46.1(34.95-65.7)$ & $50.4(37.2-70.12)$ & $47.16(34.95-70.12)$ \\
\hline Age $[$ years] & $35.7(18-59)$ & $40.01(22-62)$ & $38.2(18-62)$ \\
\hline
\end{tabular}

Table II. Primary surgery

\begin{tabular}{|ccccc|}
\hline LAGB & VGB & LGCP & Jejuno-ileal bypass & Total \\
\hline $9(1.59 \%)$ & $2(0.35 \%)$ & $3(0.53 \%)$ & $2(0.35 \%)$ & $16(2.83 \%)$ \\
\hline
\end{tabular}

$L A G B$ - laparoscopic adjustable gastric banding, VGB - vertical gastric banding, LGCP - laparoscopic greater curvature placation 
itored and they underwent contrast radiography. In all the patients, the performed procedures were recorded on video.

\section{Results}

The procedure was performed laparoscopically in 565 patients. There was no need for conversion, and neither intraoperative nor perioperative deaths within $24 \mathrm{~h}$ after the surgery were noted.

\section{Intra- and perioperative complications}

Minor bleeding during the preparation of the major curvature, stapling and applying the continuous suture was controlled by means of hemostatic clips. After dissection of the short blood vessels in the gastric fundus region, 44 patients $(7.79 \%)$ developed infarcts of the posterior pole of the spleen, manifested by color change of the splenic parenchyma (Photo 1 ). There were no cases of intraoperative gastric wall injury or perforation, damage to the spleen or esophagus, or leaks from the suture line during the intraoperative leak test.

In 1 woman $(0.18 \%)$, a $5 \mathrm{~mm}$ segment of the kinked calibration tube was sutured with the stapler during the procedure. It resulted in the necessity of resection of a gastric wall fragment, and taking into account the high risk of stomach narrowing at that site, the procedure was switched to Roux en $Y$ gastric bypass with the resection of the stomach fragment sutured to the probe.

Another female patient (0.18\%) developed tachycardia (up to 140/') and hypotension (90/40 mm Hg), and a $100 \mathrm{ml}$ blood-streaked discharge from the drain was noted $6 \mathrm{~h}$ after the surgery. We decided to perform a repeat laparoscopy. The procedure revealed $2000 \mathrm{ml}$ of blood with clots inside of the peritoneal cavity, which were removed by suction. Bleeding originated from a small arterial vessel in

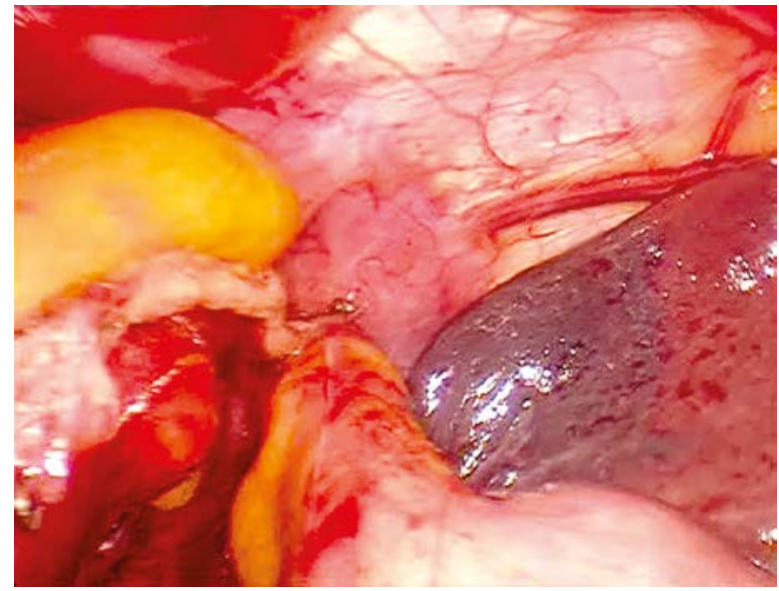

Photo 1. Infarcts of the posterior pole of the spleen

the splenodiaphragmatic ligament in the vicinity of the superior pole, and was controlled by a hemostatic clip. The drain was reinserted, the patient received blood preparations to compensate for the blood loss, and the further postoperative course was uneventful. The intraoperative complications are presented in Table III.

\section{Early postoperative complications}

Gastric leakages developed in 8 patients (1.42\%). In 2 of them $(0.35 \%)$, they manifested on the first postoperative day, accompanied by tachycardia, epigastric pain with the Blumberg sign and the presence of sputum in the drain. Oral administration of methylene blue and radiography with oral water-based contrast (Gastrografin) confirmed the presence of a leakage. In the remaining 6 patients (1.06\%), the symptoms of leakage such as epigastric pain and increased heart rate were observed on the $5^{\text {th }}(3$ patients $-0.53 \%)$ and $6^{\text {th }}(3$ patient $-0.53 \%)$ postoperative day. It should be noted that the leak test with methylene blue performed earlier did not

Table III. Intraoperative adverse events

\begin{tabular}{|lcc|}
\hline Complication & $\begin{array}{c}\text { Incidence, } \\
n(\%)\end{array}$ & Management method \\
\hline Minor bleeding without any hemodynamic significance & $47(8.32)$ & Hemostatic clip \\
\hline Splenic infarct & $14(7.79)$ & Observation of the patient \\
\hline Calibration probe suturing & $80(14.16)$ & Resection of a fragment of the gastric wall, \\
procedure change to LRYGB
\end{tabular}

LRYGB - laparoscopic Roux en Y gastric bypass 
show any signs of leakage. The case of a female patient, who reported sudden, severe epigastric pain (resembling pain due to perforation of a gastric ulcer) on the $6^{\text {th }}$ postoperative day before discharge from the hospital (1 day after the leak test with methylene blue and removal of the drain) requires special attention. She developed tachycardia and her temperature was increased. Contrast abdominal radiography performed on that day failed to reveal leakage. The patient remained at the hospital, and total parenteral nutrition (TPN) was instituted. The leakage site, located at the gastroesophageal junction, was visualized in radiography on the next day.

\section{Late postoperative complications}

On the $8^{\text {th }}$ postoperative day, symptoms of acute peptic ulcer located in the gastric body and causing complete obstruction of the gastric lumen were observed in 1 patient $(0.18 \%)$. The diagnosis was established based on the upper GIT endoscopy. She received TPN and proton pump inhibitors, and the symptoms subsided after a few days. In 3 patients (0.53\%), abscesses of the splenic regions were diagnosed on the $9^{\text {th }}, 10^{\text {th }}$ and $14^{\text {th }}$ postoperative day, resulting in pain, tachycardia, and hectic fever. Computed tomography (CT) with oral and intravenous contrast was performed. In all these patients laparoscopy was done. Conversion was necessary in 2 cases. In both cases, the abscess was localized between the posterior pole of the spleen, the esophagus and the stomach. Purulent matter was removed from the abdominal cavity, it was drained, the material for microbiological investigations was collected, and the tightness of sutures was checked with methylene blue administered via the tube - no signs of leak were detected. The TPN was instituted. In one case, complete regression of the abnormalities was observed within one week and the patient was discharged home in good general condition. The other patients developed signs of saliva leakage appearing in the abdominal drain 23 days after the reoperation. All the leakages affected the gastroesophageal junction and were located in the initial segment of the gastric suture.

Three months after the surgery, 1 patient $(0.18 \%)$ developed a painful, fluctuating nodule at the site of insertion of one of the trocars $(10 \mathrm{~mm})$. After incision, a small amount of purulent discharge was obtained. After a few days, signs of external leakage, whose internal opening was situated at $1 / 2$ of the gastric suture, were observed in that location. After 4 weeks, the leakage healed spontaneously.

\section{Management of leakages}

The first leakage was noted in the $51^{\text {st }}$ patient operated on with sleeve gastrectomy technique. The first 3 patients with leakages were re-operated on with laparotomies; the leak site was identified, the leakage opening was closed with sutures and the area was drained. The TPN was instituted along with two wide-spectrum antibiotics. Within 1 to 2 days after the procedure, the signs of a leak became apparent again in all 3 patients. The surgery was repeated and the leakage was sutured again. The symptoms of leakages recurred within the next $24 \mathrm{~h}$. All patients from that group died within the 7 following days because of systemic inflammatory response syndrome (SIRS). In the next leakage cases, TPN was instituted, and USG- or CT-guided drainage of the affected area was applied. In 4 patients, the gastroesophageal prostheses used were a self-inflating UItraflex Esophageal NG Stent System-Boston Scientific, manufactured by Boston Scientific and a silicone one manufactured by Rusch. The superior portion of the stent was positioned in the esophagus above the leakage opening, and the inferior one in the stomach below the leakage. After 8 weeks, during which 1 of the patients was hospitalized, and the others were treated on an outpatient basis, the stents were removed and no signs of leakages were detected. Then, endoscopic management of leakages was attempted in further cases - initially by closing the leakage with Tri-clip type vascular clips - without effect; next, the over-the-scope-clip (OTSC) endoscopic closure system was used - with only temporary (1-day) leakage closure effect and subsequent recurrence of the symptoms. One female patient with a primary perisplenic abscess developed an external leakage, which, after 60 days, when the infection symptoms had subsided and a rigid canal had been formed, allowed a change of procedure to Roux en $Y$ gastric bypass with esophago-intestinal anastomosis and resection of the portion of the stomach containing the leakage. Another patient, with a persistent leakage, fed parenterally, despite complete supplementation of vitamins (particularly those from the B group), electrolytes, and microelements, developed encephalopathy of unknown etiology, and died as a result of it on the $78^{\text {th }}$ day after the first procedure. 
Table IV. Serious postoperative complication types

\begin{tabular}{|lc|}
\hline Complication & $N(\%)$ \\
\hline Gastric fistula & $11(1.95)$ \\
\hline Acute peptic ulcer & $1(0.18)$ \\
\hline Abscess in the posterior splenic pole region & $3(0.53)$ \\
\hline $\begin{array}{l}\text { Splenodiaphragmatic ligament bleeding } \\
\text { with hemorrhagic shock }\end{array}$ & $1(0.18)$ \\
\hline Total & $16(2.83)$ \\
\hline
\end{tabular}

Table V. Causes of death

\begin{tabular}{|lc|}
\hline Cause & $N(\%)$ \\
\hline $\begin{array}{l}\text { Multiorgan failure in the course of sepsis } \\
\text { that developed as a result of a fistula }\end{array}$ & $3(0.53)$ \\
\hline $\begin{array}{l}\text { Encephalopathy in a female patient treated } \\
\text { for fistula (TPN) }\end{array}$ & $1(0.18)$ \\
\hline Myocardial infarction & $1(0.18)$ \\
\hline Total & $5(0.88)$ \\
\hline
\end{tabular}

Table VI. The body weight reduction effects

\begin{tabular}{|ccccc|}
\hline $\begin{array}{c}\text { After } 6 \text { months } \\
(n=521) \\
\text { \%EWL }\end{array}$ & $\begin{array}{c}\text { After 12 months } \\
(n=407) \\
\text { \%EWL }\end{array}$ & $\begin{array}{c}\text { After 24 months } \\
(n=295) \\
\% E W L\end{array}$ & $\begin{array}{c}\text { After 36 months } \\
(n=199) \\
\% E W L\end{array}$ & $\begin{array}{c}\text { After } 60 \text { months } \\
(n=93) \\
\% E W L\end{array}$ \\
\hline 58.9 & $\begin{array}{c}72.9 \\
(21.3-95.5)\end{array}$ & 67.1 & 69.6 & 73.1 \\
\end{tabular}

\section{Long-term complications}

Among the patients coming for follow-up visits, $37.9 \%$ reported heartburn. Pharmacological treatment caused regression of the symptoms in $78.4 \%$ of the group, and alleviation in $18.5 \%$. There was no improvement despite treatment in $3.1 \%$ of the patients.

In total, serious postoperative complications occurred in 16 patients $(2.83 \%)$. Their types are presented in Table IV.

Five of the patients ( $0.88 \%)$ died. In 3 of them $(0.53 \%)$, the death was due to SIRS in the course of leakage, in 1 female patient $(0.18 \%)$ due to encephalopathy of unknown etiology, and in 1 male patient $(0.18 \%)$ due to extensive myocardial infarction on the $4^{\text {th }}$ postoperative day (Table V).

The patients came for follow-up visits at 6-month intervals. They underwent blood tests, completed a questionnaire concerning weight loss and concomitant diseases, and radiography was performed. To date, it has been possible to evaluate the percentage of excess weight loss (\%EWL) after 60 months in 93 patients. The body weight reduction effects are presented in Table VI.

For all the patients with complications, video recordings of performed procedures were analyzed in order to determine the etiology.

\section{Discussion}

The results concerning intra- and postoperative complications obtained by us are consistent with the literature data indicating that sleeve gastrectomy is a safe procedure, and is easy to perform for a surgeon experienced in laparoscopic techniques [3-9]. During the surgery, mild complications are possible, associated primarily with bleeding from the greater curvature and from the gastric incision site. In our experience, blood loss is negligible and easy to manage with hemostatic clips, and requiring no transfusions. It is interesting that despite dissection performed in the vicinity of the spleen, often under extremely difficult technical conditions, there are practically no descriptions of splenic injuries in the literature.

The most serious complication that develops after LSG is gastric leakage. The incidence of this complication in our materials (1.42\%) is consistent with the data reported by other authors. If we assume that one of the perisplenic abscesses was initially a missed gastric leak, then the overall gastric leak rate in our study will be $1.59 \%$, which corresponds to available bibliographical data [2, 5, 10-14]. Unfortunately, in our material 3 patients died because of leakages and subsequent septic complications. They were the first 3 leakage patients in our practice, with early postoperative leakages $\left(1^{\text {st }}, 2^{\text {nd }}\right.$ and $5^{\text {th }}$ postoperative day). The patients underwent reoperations involving suture placement at the leakage site, with recurrence of the leakage as the outcome in all the cases. Despite drainage of the leakage region, antibiotic therapy, parenteral feeding and intensive care, the patients died due to septic symptoms. There- 
fore, we support the opinion of some authors that attempts to suture the leak are bound to be unsuccessful, as its cause is not associated with the suture placement technique, but with different etiology [11, $13,15,16]$. In the general opinion, the reason for leakages may be the use of staplers with inappropriate staple height, errors (burns of the gastric wall) associated with use of the dissecting instruments (Ligasure, harmonic knife), or increased pressure within the formed gastric sleeve. There are no unequivocal assessments concerning the size of the calibration tube used (from $32 \mathrm{Fr}$ to $60 \mathrm{Fr}$ ), although many authors claim that the wider the tube, the lower probability of leakage development [17-23]. Bariatric surgeons' discussions on the consensus on sleeve gastrectomy complications management haven't yielded any equivocal opinion on the subject yet [24-27]. There is also no unequivocal opinion concerning additional safeguarding of the gastric incision site by overlays or sutures applied manually to reinforce the mechanical suture, or the use of tissue adhesive and the effect of such measures on the incidence of leakages. Many authors believe that the incidence of intra- and postoperative bleeding can be reduced, but it does not affect the incidence of leakages $[15,28,29]$. We also support this standpoint. Based upon our material, we think that in the case of experienced surgical teams, inappropriate selection of staplers is difficult to blame, whereas accidental or unnoticed burns of the gastric wall might be a more likely cause of leakage development. The question is, how can the fact that all fistulas are located at the gastroesophageal junction in the His angle region be explained? It may be attributed to the pressure increase within the gastric sleeve created during surgery. Bellanger and Greenway report in their paper that with appropriate sleeve width $(50 \mathrm{Fr}$ ) in the His angle region, using $34 \mathrm{Fr}$ width for the remaining portion of the stomach, they had no leak complications among 567 procedures performed [28]. Using that technique (with particular attention paid to the sleeve width in the His angle region), no leakages were noted in our material as well. It is, however, striking that patients with gastric tubes, which should have prevented hypertension inside the sleeve, also developed leakages.

The analysis of video materials from the surgery, performed always in the case of complications, did not allow us to identify the causes of complications. Neither narrowing in the vicinity of the His angle, nor burns in that area during its dissection led to detection. In only 1 case, during application of the reinforcing suture over the mechanical suture line, a hematoma was formed in the proximal $1 / 3$ of the anterior gastric wall, which theoretically might have caused pressure increase within the sleeve.

The analysis of video materials also allowed us to confirm that the 3 patients diagnosed with abscesses of the splenic angle region developed infarcts of that part of the spleen during the primary procedure. In our opinion, such a phenomenon, described occasionally [30-32], and observed in our material in $7.79 \%$ of patients, may cause complications, and possibly some (late) leakages, secondary to the abscess. It is possible that the development of the spleen abscess is related to its infarction due to cutting of the short blood vessels, and the abscess is the secondary cause of the suture's leakage.

As far as the management of leakages is concerned, we can state on the basis of our own experience that instant institution of management and cooperation between specialists play an important role here. We do not recommend attempts to suture the leaks, as they usually lead to failure. The natural instinct of every surgeon working with the digestive system is to sew the leak. Unfortunately, in the case of sleeve gastrectomy (SG) complications, this technique does not prove to be appropriate. Firstly, the sutures are applied to tissues affected by an inflammatory process, in an infected environment; second$l y$, the procedure does not eliminate the cause of the problem, but only attempts (usually without success) to deal with the symptoms. Secondary suture placement proved unsuccessful in our experience. If laparoscopy or laparotomy is performed, it should be done in order to drain the leakage region effectively, lavage the septic discharge, if necessary, collect material for bacterial culture, and form an ileostomy for nutrition.

We did not observe any distal sleeve stenoses in patients with gastric leaks, and there was no pylorospasm; hence neither recommended endoscopic sleeve dilations nor pyloroplasties were carried out. Many authors emphasize the relationship between the gastric leak rate and small diameter of calibration catheters ( $<40 \mathrm{Fr}$ ), but no correlation between catheter diameter and percentage of gastric leaks was observed whatsoever. International regulations for this procedure leave the operator a wide margin of autonomy with regard to procedure parameters; hence prospective studies with catheter diameter 
randomization will allow accurate standards for this procedure to be established [22, 23].

Wide-spectrum, multi-drug antibiotic therapy is also necessary, accompanied by parenteral or enteral nutrition with substitution of microelements and vitamins. Placement of a coated prosthesis is a very good solution, allowing oral nutrition of the patient and not always requiring hospitalization. Migration of the prosthesis is an important problem, especially when the diameter of the formed sleeve is different from that of the esophagus (which favors the stent migration even at the moment of its placement); an interesting solution to the migration problem is clipping the upper portion of the prosthesis to the esophageal mucosa $[3,11,16,17,33-36]$.

Endoscopic OTSC system clips are a good method of leak closure, which allow closure even of wide leakages. Performing the procedure as early as possible, before extensive inflammatory infiltration, edema and engorgement of the tissue develops, which was probably the reason for our failures with this method, is necessary for success $[6,37]$.

Surgical treatment involving the switch of sleeve to Roux en $Y$ gastric bypass or use of the Roux en $Y$ limb is possible in our opinion after regression of the local signs of inflammation; otherwise, new complications should be expected [38].

In many cases LSG is performed as a repair procedure after other bariatric techniques. In our material, the reason for all reoperations was the lack of a slimming effect, or recurrence of obesity after previous success. In contrast to the available literature, we observed no complications in this group of patients [9].

The effects observed in our patients with respect to overweight reduction are better than those usually reported in the literature. This may be due to careful qualification and selection of patients before surgical treatment $[2,5,6,39,40]$.

Four patients died because of gastric leakages. Three of them, despite intensive treatment, developed symptoms of sepsis, which was the cause of death. We would like to emphasize that they were the first leakage patients in our material, all operated on in an attempt to close the leakage, which failed. The fourth patient, treated with TPN, developed fatal encephalopathy of unknown etiology. The patient was appropriately supplemented with vitamins and microelements, under the guidance of a neurologist and a dietician. According to neurologists, the cause of the encephalopathy was mainly due to electrolyte disorders. Despite these measures, the patient developed encephalopathy, the cause of which could not be found in spite of numerous investigations.

The comparison of the incidence of leakages in consecutive patients with the learning curve indicates that the first leakage appeared in the $51^{\text {st }}$ patient operated on by us, then they were noted up to the $204^{\text {th }}$ patient, whereas currently we do not observe any more serious complications among our patients.

Taking into account the above-mentioned data regarding morbidity and mortality rates, it should be considered why patients choose sleeve gastrectomy over gastric bypass when asked. Comparable if not lower morbidity of the gastric bypass procedure should be the culprit of its domination. In our opinion, the leading factor determining the patient's choice appears to be firstly a kind of "trend", originating in the fact that sleeve gastrectomy is a novelty attracting the crowd, and, secondly, the fact that SG affects the patient's metabolism less than the gastric bypass does, not requiring vitamin supplementation (especially from the B group, as reported in the literature). Moreover, it appears easier than Roux en $Y$ gastric bypass (RYGB) to the performing physician, which may contribute to the subconscious promotion of this method over RYGB during preoperative consultation with patients.

\section{Conclusions}

It can be stated that sleeve gastrectomy is an effective and relatively safe procedure. We cannot establish unequivocally how the most severe complication, i.e. gastric leakage, develops.

The treatment of patients who develop leakages involves predominantly conservative management (TPN, antibiotherapy) and drainage, as well as endoscopic methods, such as prostheses, clips, and tissue adhesives. Classic surgical treatment with attempts to suture the leakage is not recommended.

In our opinion, infarctions of the superior pole of the spleen, as a potential cause of abscesses in that region and secondary leakages, deserve particular attention.

\section{References}

1. Alverdy JC, Prachand V. Stapling and sleeve: science and myths. Surg Laparosc Endosc Percutan Tech 2010; 20: 141-5. 
2. Angrisani L, Cutolo PP, Buchwald JN, et al. Laparoscopic reinforced sleeve gastrectomy: early results and complications. Obes Surg 2011; 21: 783-93.

3. Aretxabala X, Leon J, Wiedmaier G, et al. Gastric leak after sleeve gastrectomy: analysis of its management. Obes Surg 2011; 21: 1232-7.

4. Frezza EE, Reddy S, Gee LL, Wachtel MS. Complications after sleeve gastrectomy for morbid obesity. Obes Surg 2009; 19: 684-7.

5. Himpens J, Dobbeleir J, Peeters G. Long-term results of laparoscopic sleeve gastrectomy for obesity. Ann Surg 2010; 252: 319-24.

6. Jacobs M, Bisland W, Gomez E, et al. Laparoscopic sleeve gastrectomy: a retrospective review of 1- and 2-year results. Surg Endosc 2010; 24: 781-5.

7. Lalor PF, Tucker ON, Szomstein S, Rosenthal RJ. Complications after laparoscopic sleeve gastrectomy. Surg Obes Relat Dis 2008; 4: 33-8.

8. Moy J, Pomp A, Dakin G, et al. Laparoscopic sleeve gastrectomy for morbid obesity. Am J Surg 2008; 196: e56-9.

9. Nocca D, Krawczykowsky D, Bomans B, et al. A prospective multicenter study of 163 sleeve gastrectomies: results at 1 and 2 years. Obes Surg 2008; 18: 560-5.

10. Burgos AM, Braghetto I, Csendes A, et al. Gastric leak after laparoscopic-sleeve gastrectomy for obesity. Obes Surg 2009; 19 : 1672-7.

11. Casella G, Soricelli E, Rizzello M, et al. Nonsurgical treatment of staple line leaks after laparoscopic sleeve gastrectomy. Obes Surg 2009; 19: 821-6.

12. Csendes A, Braghetto I, Leon P, Burgos AM. Management of leaks after laparoscopic sleeve gastrectomy in patients with obesity. J Gastrointest Surg 2010; 14: 1343-8.

13. Lacy A, Ibarzabal A, Pando E, et al. Revisional surgery after sleeve gastrectomy. Surg Laparosc Endosc Percutan Tech 2010; 20: 351-6.

14. Triantafyllidis G, Lazoura O, Sioka E, et al. Anatomy and complications following laparoscopic sleeve gastrectomy: radiological evaluation and imaging pitfalls. Obes Surg 2011; 21: 473-8.

15. Marquez MF, Ayza MF, Lozano RB, et al. Gastric leak after laparoscopic sleeve gastrectomy. Obes Surg 2010; 20: 1306-11.

16. Tan JT, Kariyawasam S, Wijeratne T, Chandraratna HS. Diagnosis and management of gastric leaks after laparoscopic sleeve gastrectomy for morbid obesity. Obes Surg 2010; 20: 403-9.

17. Gagner M. Leaks after sleeve gastrectomy are associated with smaller bougies: prevention and treatment strategies. Surg Laparosc Endosc Percutan Tech 2010; 20: 166-9.

18. Kasalicky M, Dolezel R, Vernerova E, et al. Laparoscopic sleeve gastrectomy without over-sewing of the staple line is effective and safe. Videosurgery Miininv 2014; 9: 46-52.

19. Kaska Ł, Proczko M, Stefaniak T, J et al. Redesigning the process of laparoscopic sleeve gastrectomy based on risk analysis resulted in 100 consecutive procedures without complications. Videosurgery Miniinv 2013; 8: 289-300.

20. Paluszkiewicz R, Kalinowski P, Wróblewski T, et al. Prospective randomized clinical trial of laparoscopic sleeve gastrectomy versus open Roux-en-Y gastric bypass for the management of patients with morbid obesity. Videosurgery Miniinv 2012; 7: 225-32.
21. Yehoshua RT, Eidelman LA, Stein M, et al. Laparoscopic sleeve gastrectomy: volume and pressure assessment. Obes Surg 2008; 18: 1083-8.

22. Parikh M, Issa R, Mccrillis A, et al. Surgical strategies that may decrease leak after laparoscopic sleeve gastrectomy. Ann Surg 2013; 257: 231-7.

23. Yuval JB, Mintz Y, Cohen MJ, et al. The effects of bougie caliber on leaks and excess weight loss following laparoscopic sleeve gastrectomy. Is there an ideal bougie size? Obes Surg 2013; 23: 1685-91.

24. Deitel M, Crosby RD, Gagner M. The first international consensus summit for sleeve gastrectomy (SG), New York City, October 25-27, 2007. Obes Surg 2008; 18: 487-96.

25. Deitel M, Gagner M, Erickson AL, Crosby RD. Third International Summit: current status of sleeve gastrectomy. Surg Obes Relat Dis 2011; 7: 749-59.

26. Gagner M, Deitel M, Kalberer TL, et al. The Second International Consensus Summit for sleeve gastrectomy, March 19-21, 2009. Surg Obes Relat Dis 2009; 5: 476-85.

27. Gagner M, Deitel M, Erickson AL, et al. Survey on laparoscopic sleeve gastrectomy (LSG) at the Fourth International Consensus Summit on sleeve gastrectomy. Obes Surg 2013; 23: 2013-7.

28. Bellanger DE, Greenway FL. Laparoscopic sleeve gastrectomy, 529 cases without a leak: short-term results and technical considerations. Obes Surg 2011; 21: 146-50.

29. Kasalicky M, Michalsky D, Housova J, et al. Laparoscopic sleeve gastrectomy without an over-sewing of the staple line. Obes Surg 2008; 18: 1257-62.

30. Dhanasopon AP, Lewis CE, Folek JM, et al. Splenic infarct as complication of sleeve gastrectomy. Surg Obes Relat Dis 2009; 5: 626-9.

31. Lewis CE, Dhanasopon A, Dutson EP, Mehran A. Early experience with laparoscopic sleeve gastrectomy as a single-stage bariatric procedure. Am Surg 2009; 75: 945-9.

32. Michalik M, Budzinski R, Orlowski M, et al. Splenic infarction as a complication of laparoscopic sleeve gastrectomy. Videosurgery Miniinv 2011; 6: 92-8.

33. Kim Z, Kim YJ, Kim YJ, et al. Successful management of staple line leak after laparoscopic sleeve gastrectomy with vascular plug and covered stent. Surg Laparosc Endosc Percutan Tech 2011; 21: e206-8.

34. Oshiro T, Kasama K, Umezawa A, et al. Successful management of refractory staple line leakage at the esophagogastric junction after a sleeve gastrectomy using the HANAROSTENT. Obes Surg 2010; 20: 530-4.

35. Serra C, Baltasar A, Andreo L, et al. Treatment of gastric leaks with coated self-expanding stents after sleeve gastrectomy. Obes Surg 2007; 17: 866-72.

36. Trelles N, Gagner M, Palermo M, et al. Gastrocolic leakage after re-sleeve gastrectomy: outcomes after esophageal stent implantation. Surg Obes Relat Dis 2010; 6: 308-12.

37. Conio M, Blanchi S, Repici A, et al. Use of an over-the-scope clip for endoscopic sealing of a gastric leakage after sleeve gastrectomy. Endoscopy 2010; 42 Suppl 2: E71-2.

38. Baltasar A, Serra C, Bengochea M, et al. Use of Roux limb as remedial surgery for sleeve gastrectomy leakages. Surg Obes Relat Dis 2008; 4: 759-63. 
39. Bohdjalian A, Langer FB, Shakeri-Leidenmuhler S, et al. Sleeve gastrectomy as sole and definitive bariatric procedure: 5-year results for weight loss and ghrelin. Obes Surg 2010; 20: 535-40.

40. Gluck B, Movitz B, Jansma S, et al. Laparoscopic sleeve gastrectomy is a safe and effective bariatric procedure for the lower BMI (35.0-43.0 kg/m2) population. Obes Surg 2011; 21: 1168-71.

Received: 11.02.2014, accepted: 21.02.2014. 